Review began 09/21/2021 Review ended 09/28/2021 Published 10/02/2021

(c) Copyright 2021

Panda et al. This is an open access article distributed under the terms of the Creative Commons Attribution License CC-BY 4.0. which permits unrestricted use, distribution, and reproduction in any medium, provided the original author and source are credited.

\section{A Comparison Between Transcutaneous Bilirubin and Total Serum Bilirubin Levels for the Management of Jaundice in Preterm Neonates by Bland-Altman Plot}

\author{
Santosh K. Panda ${ }^{1}$, Abhinav Gaurav ${ }^{2}$, Palash Das ${ }^{3}$, Natabar Swain ${ }^{2}$, Soumini Rath ${ }^{2}$ \\ 1. Neonatology, Kalinga Institute of Medical Sciences, Bhubaneswar, IND 2. Pediatrics, Kalinga Institute of Medical
} Sciences, Bhubaneswar, IND 3. Pediatric Medicine, Kalinga Institute of Medical Sciences, Bhubaneswar, IND

Corresponding author: Soumini Rath, soumini19@gmail.com

\section{Abstract \\ Objective}

To compare the bilirubin levels measured by transcutaneous bilirubinometer and serum samples for the management of jaundice in preterm neonates.

\section{Methods}

The study was a prospective comparative observational study conducted in a tertiary care neonatal unit of Odisha from January 2019 to June 2020. All inborn and outborn neonates with a gestational age of $28^{0 / 7}$ weeks to $36^{6 / 7}$ weeks with the clinical diagnosis of neonatal jaundice were included in the study. Transcutaneous bilirubin (TcB) was estimated by Dragger jaundice meter JM-105 and simultaneously venous blood and total serum bilirubin levels (TSB) were measured by diazonium method. The comparison between $\mathrm{TcB}$ and TSB values was analyzed by direct linear correlation in scatter plot and Bland-Altman plot.

\section{Results}

A total of 167 preterm neonates $\left(66,28-33^{6 / 7}\right.$ and $\left.111,34-36^{6 / 7}\right)$, with a mean gestational age $33.55 \pm 2.36$ weeks and a mean birth weight of $1960 \pm 613$ grams, were analyzed. The mean TSB and TcB levels were 12.99 $\pm 3.47 \mathrm{mg} / \mathrm{dl}$ (min-max 4.9-18.3 mg/dl) and $14.156 \pm 4.71 \mathrm{mg} / \mathrm{dl}$ (min-max 4-20.1 mg/dL), respectively. The $\mathrm{TcB}$ is excellently correlated with TSB with a correlation coefficient of $r=0.948, p \leqslant 0.001$. The bias difference between TcB and TSB is - 1.16 (95\% CI: $2.35,-4.6) \mathrm{mg} / \mathrm{dl}$. The correlation coefficients between 28 $33^{6 / 7}$ weeks gestational age groups $(r=0.944)$ and $34-36^{6 / 7}$ gestational age $(r=0.950)$ were similar.

\section{Conclusion}

TcB is well correlated with TSB level in preterm neonates. Hence, TcB can be used for the guidance of management in these neonates.

Categories: Family/General Practice, Pediatrics, Quality Improvemen

Keywords: neonatal hyperbilirubinemia, transcutaneous bilirubin, jaundice, neonates, preterm

\section{Introduction}

Neonatal hyperbilirubinemia is a common problem with almost $50 \%$ term and $80 \%$ of preterm neonates suffering from some amount of neonatal hyperbilirubinemia [1]. Recent reports have shown the visual assessment of jaundice to be unreliable and unsafe [2]. Total serum bilirubin (TSB) level is the gold standard for the diagnosis of neonatal hyperbilirubinemia and is vital for the effective treatment of neonatal jaundice. Frequent clinical monitoring and timely serum bilirubin testing are the best strategies for the prevention of bilirubin-induced neurological damage. The TSB estimation procedure involves invasive extraction of venous blood. In premature neonates, it is especially bothering as repeated phlebotomy causes iatrogenic anemia and also increases parental anxiety [3]. In recent years, transcutaneous bilirubinometry is being used for serum bilirubin screening of neonates $>35$ weeks after 24 hours of age. The first clinically appropriate and portable transcutaneous bilirubinometer was introduced by Yamanouchi and associates working with the Minolta Camera Company [4]. In recent years, the availability of newer equipment with advanced technology is used for the management of preterm jaundice. This study was carried out to measure transcutaneous bilirubin (TcB) levels in preterm jaundiced neonates between 28 and 37 weeks of gestational age and compare their values with TSB levels.

\section{Materials And Methods}




\section{Cureus}

This study was a prospective comparative study carried out at a tertiary care neonatal center of Odisha between January 2019 and June 2020 after institutional ethical clearance. All inborn and outborn neonates with a gestational age of $28^{0-7}$ weeks to $36^{6-7}$ weeks with the clinical diagnosis of neonatal jaundice, requiring laboratory investigation for serum bilirubin, were included in the study, after taking informed consent from the parents. Neonates developing jaundice within the first 24 hours of life, and neonates who already received phototherapy treatment for neonatal jaundice and had raised direct serum bilirubin $>2$ $\mathrm{mg} / \mathrm{dl}$ from blood investigation were excluded from the study. Neonates were screened by Dragger jaundice meter JM-105 and venous blood was obtained for TSB. The TSB is measured by the diazonium method. A total of three readings of the bilirubin meter were taken over the mid sternum area and the average of three readings was recorded in "mg/dl." For the entire study period, we ensured that the instrumentations, the methodology, and the calibrators are not changed in the laboratory. A single observation of both TcB and TSB of each participant neonate was analyzed in this study. The demographic parameters gestational age, birth weight, gender, mode of delivery, neonates blood group, maternal blood group, and G6PD status were collected from the neonatal case records. Neonates were followed every 12 hours by neonatologist and jaundice was managed as per National Institute for Health and Care Excellence guidelines.

\section{Statistical analysis}

All the continuous data were presented as mean \pm standard deviation (SD) and categorical data as frequency and percentage. Pearson's correlation coefficient calculation and linear regression analysis were used to check the relationship between TcB and TSB. Bland-Altman plot was placed for qualitative assessment of variables. All the data analyses were performed using statistical software IBM SPSS version 20.0 (IBM Corp, Armonk, NY).

\section{Results}

\section{Observations and results}

A total of 167 preterm neonates were enrolled with a mean gestational age of $33.55 \pm 2.36$ weeks and a mean birth weight of $1960 \pm 613$ grams. In this study, 69 (41.3\%) were females, 66 (39.5\%) were early preterm <34 weeks, and 44 (26.3\%) were very-low-birth-weight neonates (birth weight < 1500 grams). Hyperbilirubinemia was related to blood group incompatibility in 27 (16.2\%), 21 (12.6\%) cases were G6PD deficiency, and 119 (71.25\%) were idiopathic neonatal jaundice. The mean TSB and TcB levels were $12.99 \pm 3.47 \mathrm{mg} / \mathrm{dl}$ ( $\mathrm{min}-\mathrm{max}$ $4.9-18.3 \mathrm{mg} / \mathrm{dl})$ and $14.156 \pm 4.71 \mathrm{mg} / \mathrm{dl}(\min -\max 4-20.1 \mathrm{mg} / \mathrm{dl})$, respectively.

The scatter plot of TSB versus TcB (Figure 1) shows a strong direct linear correlation. Pearson's correlation coefficient, $r=0.948$ and $\mathrm{R}^{2}=0.898$ with $\mathrm{p} \leqslant 0.001$. Simple linear regression is calculated as $\mathrm{TSB}=3.121+$ $0.698 \times$ TcB. This equation can be used for predicting TSB from TcB with a high degree of accuracy.

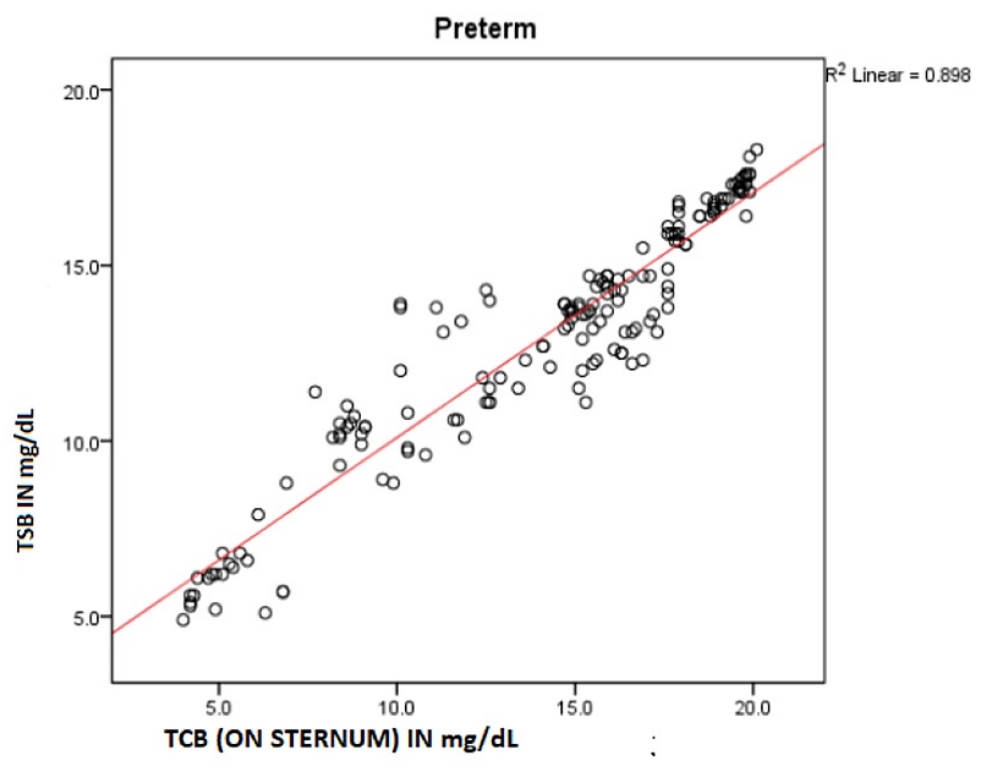

FIGURE 1: Scatter plot of TSB versus TcB $(n=167)$

TSB, total serum bilirubin; TcB, transcutaneous bilirubin 


\section{Cureus}

suggests the mean of TSB and TcB. Y-axis was plotted by the difference between TSB and TcB. The bias line indicates a difference of $1.16 \mathrm{mg} / \mathrm{dl}$ between the averages of the two variables. Analysis of mean bilirubin level showed that TSB was $1.16 \mathrm{mg} / \mathrm{dl}$ lower than the measured TcB. The majority of the data points fall within \pm 1.96 times the SD of the difference between the TSB and TcB values. This corroborates that there is a strong agreement between the two variables.

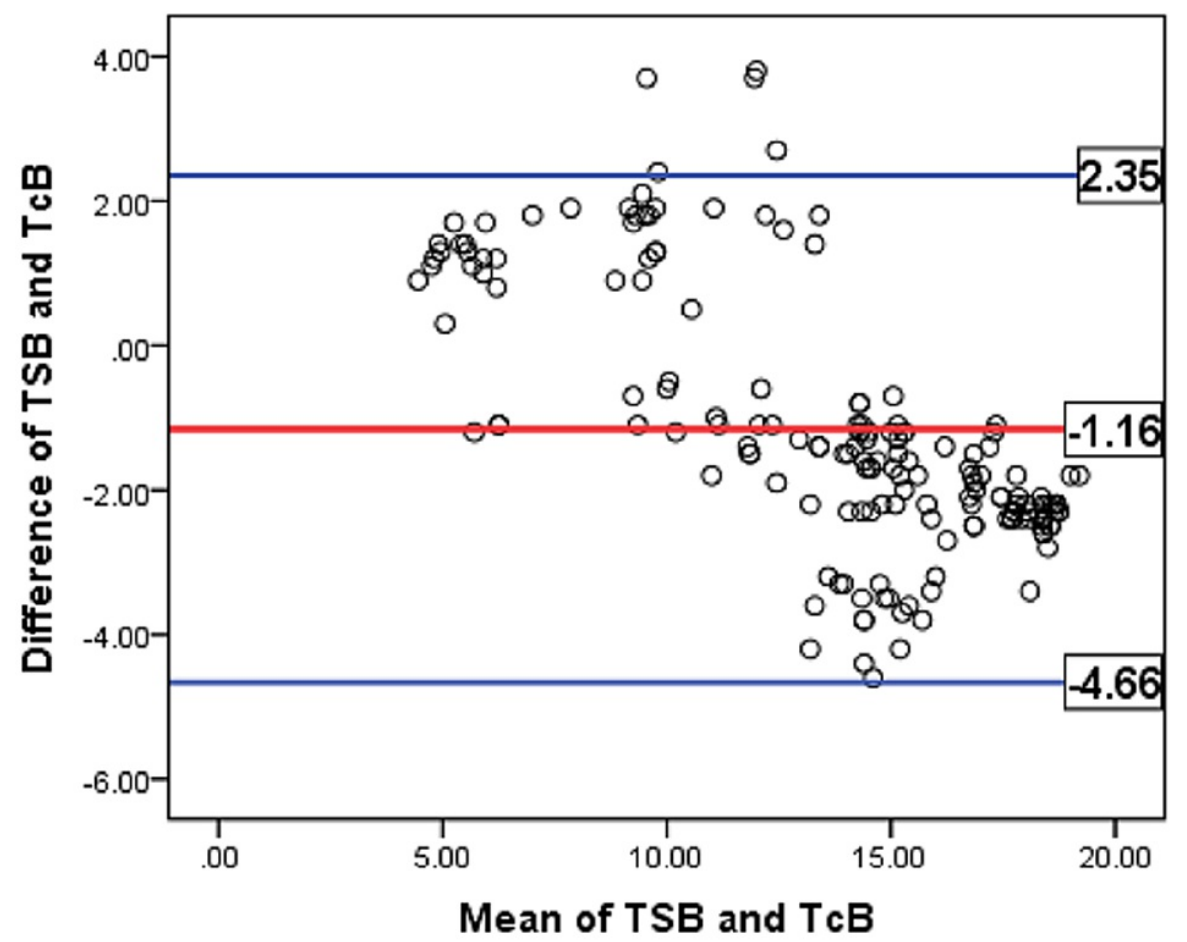

FIGURE 2: Bland-Altman plot of TSB versus TcB $(n=167)$

TSB, total serum bilirubin; TcB, transcutaneous bilirubin.

In subgroup analysis, TcB in babies $28-33^{6 / 7}$ weeks of gestation $(\mathrm{r}=0.944 ; \mathrm{p}<0.001)$ are nearly equally correlated with late preterm neonates $(r=0.950, p<0.001)$. The bias difference between TcB and TSB in lower preterm groups and late preterm groups was $1.24 \mathrm{mg} / \mathrm{dl}$ and $1.1 \mathrm{mg} / \mathrm{dl}$, respectively.

\section{Discussion}

In the present study, TcB is excellently correlated $(r=0.94, p \leqslant 0.001)$ with TSB in preterm neonates prior to phototherapy. The correlation coefficient between TcB and TSB among lower-gestational-age neonates $(\mathrm{r}=$ $0.944)$ is comparable to late-preterm neonates $(\mathrm{r}=0.950)$. In the published literature available, this is the largest series of preterm neonates with regard to estimation of TcB by JM-105, advanced equipment for noninvasive bilirubin estimation. Already two systematic reviews and meta-analyses by Nagar et al. and Shabuj et al. depict the reliability of TcB devices (JM-103 and Bilicheck) in preterm infants $[5,6]$. The TcBmeters like JM-103 or Bilicheck reliably estimated bilirubin levels in preterm infants; however, the JM-103 device exhibited better precision than the Bili Check. The JM-105 used in this study is the next generation of JM103 , with advanced features in the basic functionality, i.e. measuring probe, hardware, and software used for measurements [7]. TCB measured by JM-105 is more reliable $(\mathrm{r}=0.944)$ than JM-103 or Bilicheck (pooled correlation coefficient around 0.83 mentioned in the systematic reviews) $[5,6]$.

Similar to previous studies by Mandal et al. [8], Pendse et al. [9], Pratesi et al. [10], and Kitsommart et al. [11], a significant correlation was observed here between TcB and TSB values. The correlation coefficient of TcB and TSB in this study is incongruent to Pendse et al.'s with a similar gestational age group in the Indian preterm neonatal population using JM-105. However, in contrast to our findings, the correlation coefficient is higher in the lower gestational age group compared to higher gestational age babies [9]. In a study by Kumar et al., a better correlation of $\mathrm{TcB}$ was noticed among lower gestational age compared to higher gestational age groups, but TcB was measured here by Bilitest-2000 [12]. Negar et al. saw the maximum correlation in 33-37 weeks of gestation and birth weight more than 2500 grams in Iranian preterm neonates using JH 20-1A for TcB measurement [13]. The accuracy of TcB in preterm neonates could be modified by gestational age, racial factors, sickness of the neonate, and types of equipment. 
In the Bland-Altman plot of this study, the bias line indicated the mean difference of $1.16 \mathrm{mg} / \mathrm{dl}$ between TcB and TSB. The majority of the data points fell within \pm 1.96 times the SD of the difference between TSB and $\mathrm{TcB}$ values. This corroborates that there is a strong agreement present. The mean difference between TSB and TcB in preterm neonates before phototherapy is $0.64 \mathrm{mg} / \mathrm{dl}$ [95\%: 0.13-1.14] in Posada et al. using JM105 [14]. The slightly higher accuracy of Posada et al. could be secondary to the difference in the gestational age distribution and racial difference. Overall, TcB meter is handy, easy to carry out at the bedside, gives the result instantly, and decreases the frequency of painful pricking [15].

\section{Limitation of the study}

The study is monocentric with limited numbers of extremely-low-birth-weight neonates. It was done in clinically jaundiced preterm neonates before phototherapy rather than during phototherapy or after phototherapy to evaluate its accuracy during rebound hyperbilirubinemia.

\section{Conclusions}

The TSB level estimated by the conventional method has a significant correlation with the value of bilirubin as measured by the transcutaneous bilirubinometer in preterm neonates. The use of a transcutaneous bilirubinometer will decrease the frequency of painful phlebotomy in preterm neonates and will also decrease parental anxiety. We recommend that transcutaneous bilirubinometer can be effectively used as a screening tool to predict bilirubin levels even in premature babies and can be used to dictate management guidelines. Further studies with a larger cohort of babies, and estimating TcB after phototherapy and correlating with TSB will further help in strengthening transcutaneous bilirubinometer as a diagnostic device. As per our study, the values of TcB correlated very well with TSB measured in the laboratory by standard methods and should be used in screening and deciding therapeutic interventions in premature neonates as well.

\section{Additional Information \\ Disclosures}

Human subjects: Consent was obtained or waived by all participants in this study. Kalinga Institute of Medical Sciences Institution Ethics board issued approval KIMS/KIIT/IEC/164/2018. The study was cleared by the Institutional Review Board and Ethics Committee under the ethical clearance no KIMS/KIIT/IEC/164/2018. Animal subjects: All authors have confirmed that this study did not involve animal subjects or tissue. Conflicts of interest: In compliance with the ICMJE uniform disclosure form, all authors declare the following: Payment/services info: All authors have declared that no financial support was received from any organization for the submitted work. Financial relationships: All authors have declared that they have no financial relationships at present or within the previous three years with any organizations that might have an interest in the submitted work. Other relationships: All authors have declared that there are no other relationships or activities that could appear to have influenced the submitted work.

\section{References}

1. Akobeng AK: Neonatal jaundice. Am Fam Physician. 2005, 71:947-8.

2. Gunaseelan S, Devadas S, Pai N: Correlation of transcutaneous bilirubin and serum bilirubin concentration in term and late preterm newborns. J Clin Neonatol. 2017, 6:154-8. 10.4103/jcn.JCN_109_16

3. Committee on Fetus and Newborn and Section on Anesthesiology and Pain Medicine: Prevention and management of procedural pain in the neonate: an update. Pediatrics. 2016, $137:$ :20154271. 10.1542/peds.2015-4271

4. Yamanouchi I, Yamauchi Y, Igarashi I: Transcutaneous bilirubinometry: preliminary studies of noninvasive transcutaneous bilirubin meter in the Okayama National Hospital. Pediatrics. 1980, 65:195-202.

5. Nagar G, Vandermeer B, Campbell S, Kumar M: Reliability of transcutaneous bilirubin devices in preterm infants: a systematic review. Pediatrics. 2013, 132:871-81. 10.1542/peds.2013-1713

6. Hassan Shabuj M, Hossain J, Dey S: Accuracy of transcutaneous bilirubinometry in the preterm infants: a comprehensive meta-analysis. J Matern Fetal Neonatal Med. 2019, 32:734-41. $10.1080 / 14767058.2017 .1390561$

7. 510(k) Premarket Notification. (2020). Accessed: September 27, 2021: https://www.accessdata.fda.gov/scripts/cdrh/cfdocs/cfpmn/pmn.cfm.

8. Mandal A, Bannerji R, Ray J, Mitra M, Azad S, Basu S: Correlation between transcutaneous bilirubin estimation and total serum bilirubin estimation in neonatal hyperbilirubinemia. BLDE Univ J Health Sci. 2018, 3:36-42. 10.4103/bjhs.bjhs_6_17

9. Pendse A, Jasani B, Nanavati R, Kabra N: Comparison of transcutaneous bilirubin measurement with total serum bilirubin levels in preterm neonates receiving phototherapy. Indian Pediatr. 2017, 54:641-3. 10.1007/s13312-017-1126-y

10. Pratesi S, Boni L, Tofani L, Berti E, Sollai S, Dani C: Comparison of the transcutaneous bilirubinometers BiliCare and Minolta JM-103 in late preterm and term neonates. J Matern Fetal Neonatal Med. 2016, 29:3014-8. 10.3109/14767058.2015.1113521

11. Kitsommart R, Yangthara B, Wutthigate P, Paes B: Accuracy of transcutaneous bilirubin measured by the BiliCare device in late preterm and term neonates. J Matern Fetal Neonatal Med. 2016, 29:3641-5. $10.3109 / 14767058.2016 .1140141$ 


\section{Cureus}

12. Kumar V, Kahlon PS, Singh P, Singh K, Sharma A: Utility of transcutaneous bilirubinometer in tertiary care hospital. J Nepal Paediatr Soc. 2017, 37:72-8. 10.3126/jnps.v37i1.17117

13. Sajjadian N, Shajari H, Saalehi Z, Esphahani F, Alizadeh Taheri P: Transcutaneous bilirubin measurement in preterm neonates. Acta Med Iran. 2012, 50:765-70.

14. Costa-Posada U, Concheiro-Guisán A, Táboas-Ledo MF, et al.: Accuracy of transcutaneous bilirubin on covered skin in preterm and term newborns receiving phototherapy using a JM-105 bilirubinometer. J Perinatol. 2020, 40:226-31. 10.1038/s41372-019-0557-9

15. Briscoe L, Clark S, Yoxall CW: Can transcutaneous bilirubinometry reduce the need for blood tests in jaundiced full term babies?. Arch Dis Child Fetal Neonatal Ed. 2002, 86:F190-2. 10.1136/fn.86.3.f190 